\title{
3D Cellular Network Architecture with Drones for Beyond 5G
}

\author{
Mohammad Mozaffari ${ }^{1}$, Ali Taleb Zadeh Kasgari ${ }^{2}$, Walid Saad ${ }^{2}$, Mehdi Bennis ${ }^{3}$, and Mérouane Debbah ${ }^{4}$ \\ ${ }^{1}$ Ericsson, CA, USA, Email: mohammad.mozaffari@ericsson.com. \\ ${ }^{2}$ Wireless@VT, Electrical and Computer Engineering Department, Virginia Tech, VA, USA, Emails: $\{$ alitk, walids\}@vt.edu. \\ ${ }^{3}$ CWC - Centre for Wireless Communications, Oulu, Finland, Email: bennis@ee.oulu.fi. \\ ${ }^{4}$ Mathematical and Algorithmic Sciences Lab, Huawei France R \& D, Paris, France, and CentraleSupelec, \\ Universitè Paris-Saclay, Gif-sur-Yvette, France, Email: merouane.debbah@ @uawei.com.
}

\begin{abstract}
In this paper, a novel concept of three-dimensional (3D) cellular networks, that integrate drone base stations (droneBS) and drone users (drone-UEs), is introduced. For this new 3D cellular network architecture, a novel framework for the deployment of drone-BSs and latency-minimal cell association for drone-UEs is proposed. For drone-BSs' deployment, a tractable method based on the notion of truncated octahedron shapes is proposed that ensures full coverage for a given space with minimum number of drone-BSs. Then, an optimal 3D cell association scheme is determined such that the drone-UEs' latency, considering transmission, computation, and backhaul latencies, is minimized. In particular, using optimal transport theory, the optimal 3D cell partitions are derived according to the spatial distribution of drone-UEs and the drone-BSs' locations. Simulation results show that the proposed approach reduces the latency of drone-UEs compared to the classical cell association approach that uses a signal-to-interference-plus-noise ratio (SINR) criterion. In particular, the proposed approach yields a reduction of up to $46 \%$ in average latency compared to the SINR-based association. Also, it is shown that the proposed latency-optimal cell association improves the spectral efficiency of a 3D wireless cellular network of drones.
\end{abstract}

\section{INTRODUCTION}

Recent reports from the federal aviation administration (FAA) show that the number of unmanned aerial vehicles (UAVs), also known as drones, will exceed 7 million in 2020. From a wireless communications perspective, drones will have two key roles: aerial base stations (BSs), and user equipments (UEs) [1]-[5]. Due to their aerial nature and flexible operation, drone-BSs can support broadband communications to underdeveloped areas and provide hotspot wireless coverage during temporary events [2], [4]-[6]. Meanwhile, drones can also act as user equipments (i.e., drone-UEs) that must connect to a wireless network so as to operate. In particular, wirelessconnected drone-UEs can be used for wide range of applications such as package delivery, remote sensing, and virtual reality.

Mohammad Mozaffari joined Ericsson in July 2018. He was with Wireless@VT, Electrical and Computer Engineering Department, Virginia Tech, VA, USA, when this work was done.

This work was supported in part by the Army Research Office (ARO) under Grant W911NF-17-1-0593, in part by the US NSF under Grants AST-1506297 and CNS-1460316, and by the ERC Starting Grant MORE (Advanced Mathematical Tools for Complex Network Engineering). Dr. Bennis work was supported by Academy of Finland project CARMA, 6Genesis Flagship (grant no. 318927), the INFOTECH project NOOR, and the Kvantum Institute strategic project SAFARI.
Wireless networking with drones faces a number of challenges. For instance, for drone-BSs, key design problems include 3D deployment of drones, performance analysis, resource allocation, and cell association. For drone-UEs, there is a need for reliable and low latency communications so as to efficiently control the drones' operations. However, existing terrestrial cellular networks have been primarily designed for supporting ground users and are not able to effectively serve aerial users. In fact, terrestrial BSs may not be able to meet the low-latency and reliable communication requirements of drone-UEs due to blockage effects and the specific design of the BSs' antennas which are not suitable for supporting users at high-elevation angles. Furthermore, in areas with geographical constraints, terrestrial base stations may not be available to provide wireless service to drone-UEs. In such cases, the deployment of aerial drone-BSs is a promising opportunity for providing reliable wireless connectivity for drone-UEs. Clearly, to support the key roles of drones in wireless networking applications, there is a need for developing the novel concept of a $3 D$ cellular network that incorporates both drone-BSs and drone-UEs.

\section{A. Related Works on Drone Communications}

Recent studies on drone communications have investigated various design challenges that include performance characterization, 3D deployment, user-to-drone association, and cellularconnected UAVs. For instance, the work in [7] studied the optimal 3D deployment of UAVs for maximizing the number of covered ground users under quality-of-service constraints. In [8], the authors proposed a framework for strategic placement of drone-BSs for a large-scale ground network. However, the prior studies on deployment of UAV base stations ignore the existence of flying drone-UEs. In addition, the work in [9] proposed an algorithm for maximizing the sum-rate of ground users by joint optimization of user-to-drone-BSs association and wireless backhaul bandwidth allocations. The work in [10] proposed a novel cell association approach that maximizes the total data delivered to ground users by drone-BSs that have limited flight endurance. However, the previous works on user association in drone networks are limited to ground users and do not consider 3D aerial users. Moreover, they do not analyze latency as a key metric in 3D drone communication systems.

While there exists a number of studies on cellular-connected 
drone-UEs [4], [11], the potential use of drone-BSs for serving drone-UEs has not been considered. For example, in [11], the authors studied the coexistence of drone-UEs and ground users in cellular networks and characterized the downlink coverage performance. In [4], the authors proposed a trajectory design scheme for minimizing the mission time of a single drone-UE. However, none of these previous works [2], [4], [5], [7], [8], [10], [11], studied a 3D wireless network of co-existing aerial base stations and users (i.e., drone-BSs and drone-UEs) while addressing the deployment and latency-aware cell association problems.

\section{B. Contributions}

The main contribution of this paper is to introduce the novel concept of a fully-fledged drone-based 3D cellular network while proposing a new framework for addressing two fundamental problems of deployment and 3D cell association in such a 3D cellular network. In particular, our proposed framework includes a tractable approach for three-dimensional placement of drone-BSs and a latency-minimal 3D cell association scheme for servicing drone-UEs. For deployment, we introduce a new approach based on truncated octahedron cells that determines the locations of drone-BSs that can cover a 3D space with a minimum number of drone-BSs. For the latencyminimal 3D cell association, given the locations of drone-BSs and the distribution of drone-UEs, we find the optimal 3D cell association for which the total latency of serving droneUEs is minimized. In this case, we analytically characterize the optimal 3D cell partitions by exploiting tools from optimal transport theory. Our results show that the proposed approach significantly reduces the latency of serving drone-UEs, of up to $46 \%$, compared to classical signal-to-interference-plus-noise ratio (SINR)-based cell association approaches.

\section{SySTEM MODEL}

Consider a 3D cellular network composed of $L$ drone users, $N$ low-altitude platform (LAP) drone base stations, and a number of high-altitude platform (HAP) drones, as shown in Figure 1. We represent the sets of drone-UEs, and drone-BSs, respectively, by $\mathcal{L}$, and $\mathcal{N}$. In this aerial network, drone-BSs serve drone-UEs in the downlink, and HAP drones provide $a$ wireless backhaul connectivity for drone-BSs. Here, the deployment of drone-BSs is performed based on a 3D cellular structure which will be exposed in Section III. For backhaul connectivity, we assume that each drone-BS connects to a HAP that provides a maximum rate. Drone-BS $n$ uses transmit power $P_{n}$ and bandwidth $B_{n}$ to serve its associated flying drone-UEs. We use a spatial distribution function, $f(x, y, z)$ for drone-UEs to capture the probability that each drone-UE is present around a 3D location $\boldsymbol{v}=(x, y, z)$. Given $N$ number of drone-BSs, the space is partitioned into $N$ 3D cells each of which representing a volume that must be serviced by one drone-BS. Let $\mathcal{V}_{n}$ be a 3D space (i.e., 3D cell) associated to drone-BS $n$ that serves drone-UEs located within this cell. The average number of drone-UEs inside $\mathcal{V}_{n}$, considering $\boldsymbol{v}=(x, y, z)$, is given by:

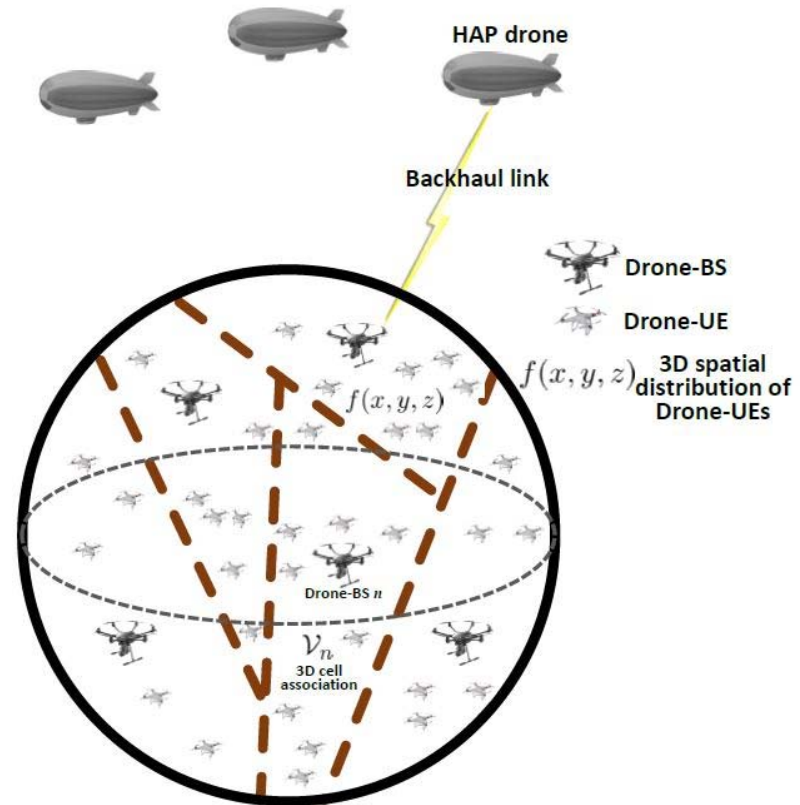

Fig. 1: The proposed 3D wireless network of drones.

$$
K_{n}=L \int_{\mathcal{V}_{n}} f(\boldsymbol{v}) \mathrm{d} \boldsymbol{v} .
$$

Each drone-BS adopts a frequency division multiple access (FDMA) technique (as done in [10]) to serve its drone-UEs. Hence, the average downlink transmission rate in drone-BS $n$ to a drone-UE located at $\boldsymbol{v}$ is:

$$
R_{n}(x, y, z)=\frac{B_{n}}{K_{n}} \log _{2}\left(1+\gamma_{n}(x, y, z)\right),
$$

where $\frac{B_{n}}{K_{n}}$ is the bandwidth needed to serve each drone-UE located in $\mathcal{V}_{n}$. Here, $\gamma_{n}(\boldsymbol{v})$ is the SINR for a drone-UE at location $(x, y, z)$ served by drone-BS $n$.

We consider the average latency in servicing drone-UEs as our main performance metric. In particular, we consider transmission latency in drone-BSs to drone-UEs communications, backhaul latency in drone-BSs to HAP drones links, and computational latency for drone-BSs that serve drone-UEs. The transmission latency for a drone-UE located at $(x, y, z)$ served by drone-BS $n$ is:

$$
\tau_{n}^{\operatorname{Tr}}\left(\boldsymbol{v}, K_{n}\right)=\frac{\beta}{R_{n}(\boldsymbol{v})},
$$

where $\beta$ is the number of bits per packet for each drone-UE.

The backhaul latency depends on the load of drone-BSs and the backhaul transmission rates. In this case, the average backhaul latency from drone-BS $n$ to its corresponding HAPdrone will be:

$$
\tau_{n}^{\mathrm{B}}\left(K_{n}\right)=\frac{\beta L \int_{\mathcal{V}_{n}} f(\boldsymbol{v}) \mathrm{d} \boldsymbol{v}}{C_{n}}=\frac{\beta K_{n}}{C_{n}},
$$

where $C_{n}$ is the maximum backhaul transmission rate for drone-BS $n$, and $\beta L \int_{\mathcal{V}_{n}} f(\boldsymbol{v}) \mathrm{d} \boldsymbol{v}$ represents the average load on drone-BS $n$.

The computation time depends on the data size (i.e., load) that must be processed at each drone-BS, and the processing 
speed. To capture the latency at drone-BS $n$, we use function $g_{n}\left(\beta K_{n}\right)$ with $\beta K_{n}$ being the total data size of the drone-BS. Therefore, the total latency experienced by a drone-UE served by drone-BS $n$ can be given by:

$$
\tau_{n}^{\mathrm{tot}}\left(\boldsymbol{v}, K_{n}\right)=\tau_{n}^{\mathrm{Tr}}\left(\boldsymbol{v}, K_{n}\right)+\tau_{n}^{\mathrm{B}}\left(K_{n}\right)+g_{n}\left(\beta K_{n}\right),
$$

Given this model, our goal is to minimize the average latency of drone-UEs by finding the optimal 3D cell associations. In particular, given the locations of drone-BSs which are deployed based on a 3D cellular structure (in Section III), and the estimated spatial distribution of drone-UEs (in Section IV), we determine the optimal $\mathcal{V}_{n}, \forall n \in \mathcal{N}$ that can lead to a minimum average latency for drone-UEs. Our 3D cell association optimization problem is given by:

$$
\min _{\mathcal{V}_{n}, n \in \mathcal{N}} \sum_{n=1}^{N}\left[\int_{\mathcal{V}_{n}} \tau_{n}^{\operatorname{Tr}}\left(\boldsymbol{v}, K_{n}\right) f(\boldsymbol{v}) \mathrm{d} \boldsymbol{v}+\tau_{n}^{\mathrm{B}}\left(K_{n}\right)+g_{n}\left(\beta K_{n}\right)\right],
$$

$$
\text { s.t. } \mathcal{V}_{l} \cap \mathcal{V}_{m}=\emptyset, \quad \forall l \neq m \in \mathcal{N}, \bigcup_{n \in \mathcal{N}} \mathcal{V}_{n}=\mathcal{V},
$$

where $K_{n}=L \int_{\mathcal{V}_{n}} f(\boldsymbol{v}) \mathrm{d} \boldsymbol{v}$ which depends on the 3D cell association, and $\mathcal{V}^{n}$ is the entire considered space in which drone-UEs can fly. Constraints in (7) ensure that the 3D association spaces are disjoint and their union covers the considered space $\mathcal{V}$.

\section{Three-Dimensional DePloyment of Drone-BSS: A TRUNCATED OCTAHEDRON STRUCTURE}

Here, we propose a framework for the 3D deployment of drone-BSs. In particular, we use the notion of truncated octahedron shapes to perform a 3D cellular network planning.

In traditional ground cellular networks, hexagonal cell shapes are used while deploying base stations. This is due to the fact that, a 2D space can be fully covered (i.e., without any gaps) by non-overlapping hexagons. Inspired by $2 \mathrm{D}$ cellular networks, we propose a framework for the deployment of a 3D cellular network. In three dimensions, the regular polyhedron geometric shapes that can tessellate the space (i.e., fill the 3D space entirely) include cube, hexagonal prism, rhombic dodecahedron, and truncated octahedron [12]. Among these 3D shapes, the truncated octahedron is the closest approximation of a sphere. Moreover, the number of polyhedron required for completely covering a 3D space is minimized by adopting the truncated octahedron [12]. Therefore, in our model, we use the truncated octahedron structure for deploying the drone-BSs.

The truncated octahedron is a $3 \mathrm{D}$ polyhedron with regular polygons faces, as see in Fig. 2. The truncated octahedron can tessellate the three-dimensional Euclidean space. In other words, the 3D space can be completely filled with multiple copies of the truncated octahedron without any overlap. We exploit this feature of the truncated octahedron in our 3D cellular network deployment with drone-BSs.

To deploy drone-BSs, we first completely fill the given space with an arrangement of multiple truncated octahedron cells. Then, we place each drone-BS at the center of each truncated

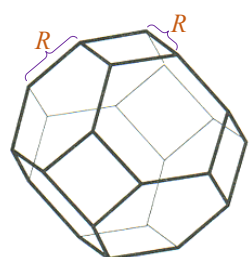

Fig. 2: Truncated octahedron in 3D.

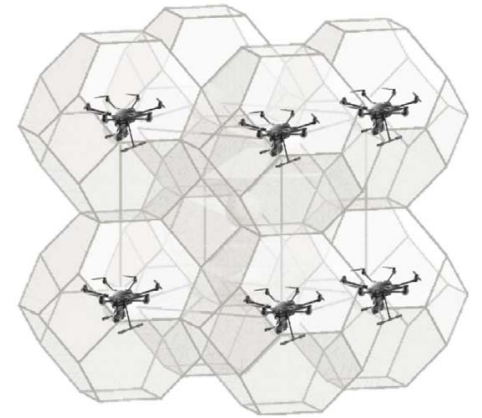

Fig. 3: Deployment of drone-BSs based on truncated octahedron cells.

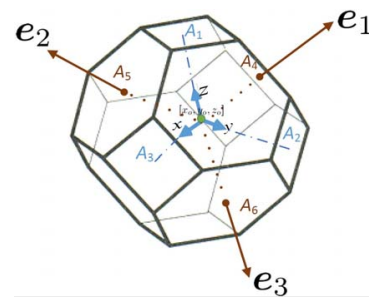

Fig. 4: Coordinate systems in drone-BSs deployment.

octahedron, as illustrated in Fig. 3. Our proposed deployment approach can ensure full coverage for a given 3D space and is tractable, easy to implement, and facilitates 3D frequency reuse. For this 3D architecture, the drone-BS deployment (i.e., optimal drone-BS locations) is characterized next.

Theorem 1. The three-dimensional locations of drone-BSs in the proposed 3D cellular network are given by:

$\boldsymbol{P}_{\{a, b, c\}}=\left[x_{o}, y_{o}, z_{o}\right]+\sqrt{2} R[a+b-c,-a+b+c, a-b+c]$,

where $a, b, \quad c$ are integers chosen from set $\{\ldots,-2,-1,0,1,2, \ldots\}$, and $R$ is the edge length of the considered truncated octahedrons. $\left[x_{o}, y_{o}, z_{o}\right]$ is the Cartesian coordinates of a given reference location (e.g., center of a specified space).

Proof. We first create a 3D lattice of truncated octahedrons whose centers are occupied by drone-BSs. Hence, to determine the locations of drone-BSs, we need to find the center of truncated octahedrons. Let $\left[x_{o}, y_{o}, z_{o}\right]$ be the center of the first truncated octahedron in the Cartesian coordinate with $x, y$, and $z$ directions being perpendicular to square faces $A_{3}, A_{2}$, and $A_{1}$ as shown in Figure 4. We find a new coordinate system whose integer coordinates are the centers of the truncated octahedrons. By moving, in integer value steps, along the axes of this coordinate systems, we can reach the centers of the truncated octahedrons. We consider a coordinate system whose axes $\left(\boldsymbol{e}_{1}, \boldsymbol{e}_{2}, \boldsymbol{e}_{3}\right)$ are vertically outward the hexagonal faces, $A_{4}, A_{5}$, and $A_{6}$. Now, we find the Euclidean length of each 
unit axis of this coordinate system. The distance between the center of the truncated octahedron and each hexagonal face is $R \sqrt{6} / 2$ [13]. Therefore, the distance between $\left[x_{o}, y_{o}, z_{o}\right]$ to the center of an adjacent truncated octahedron connecting to face $A_{4}$ is $R \sqrt{6}$. As a result, each axis unit must be $2 R \sqrt{6}$. Clearly, the centers of the truncated octahedrons in the 3D lattice are the integer coordinates of the $\left(\boldsymbol{e}_{1}, \boldsymbol{e}_{2}, \boldsymbol{e}_{3}\right)$ coordinate system. Hence, the 3D location of each drone-BS can be represented by a triple $(a, b, c)$ with $a, b$, and $c$ being integers. The position of a drone-BS obtained by $\{a, b, c\}$ is given by:

$$
\boldsymbol{P}_{\{a, b, c\}}=a \boldsymbol{e}_{1}+b \boldsymbol{e}_{2}+c \boldsymbol{e}_{3} .
$$

To represent $\boldsymbol{P}_{\{a, b, c\}}$ in Cartesian coordinates, we find the projections of $\boldsymbol{e}_{1}, \boldsymbol{e}_{2}$, and $\boldsymbol{e}_{3}$ on the $x, y$, and $z$ axes. With some geometric calculations and using the fact that the Dihedral angle (i.e., angle between two intersecting planes) between the adjacent square face and hexagonal face is $\cos ^{-1}\left(\frac{-1}{\sqrt{3}}\right)$ [13], we obtain:

$$
\left\{\begin{array}{l}
\boldsymbol{e}_{1}=R \sqrt{6}\left(\frac{-1}{\sqrt{3}} \boldsymbol{x}+\frac{1}{\sqrt{3}} \boldsymbol{y}+\frac{1}{\sqrt{3}} \boldsymbol{z}\right), \\
\boldsymbol{e}_{2}=R \sqrt{6}\left(\frac{1}{\sqrt{3}} \boldsymbol{x}+\frac{-1}{\sqrt{3}} \boldsymbol{y}+\frac{1}{\sqrt{3}} \boldsymbol{z}\right), \\
\boldsymbol{e}_{3}=R \sqrt{6}\left(\frac{1}{\sqrt{3}} \boldsymbol{x}+\frac{1}{\sqrt{3}} \boldsymbol{y}+\frac{-1}{\sqrt{3}} \boldsymbol{z}\right) .
\end{array}\right.
$$

Using (8) and (9), the 3D locations of drone-BSs with respect to the reference position $\left[x_{o}, y_{o}, z_{o}\right]$, will be:

$\boldsymbol{P}_{\{a, b, c\}}=\left[x_{o}, y_{o}, z_{o}\right]+\sqrt{2} R[a+b-c,-a+b+c, a-b+c]$.

Using Theorem 1, we can find the 3D coordinates of droneBSs deployed at the centers of truncated octahedrons. Moreover, Theorem 1 allows determining the frequency reuse factor and interfering drone-BSs in the proposed 3D network.

In summary, our approach for 3D deployment of droneBSs is as follows. We deploy the first drone-BS as a reference cell in a specified space of interest. Then, using our truncated octahedron model with parameter $R$, we use Theorem 1 to find the locations of other drone-BSs with respect to the reference cell. This results in a truncated octahedron tessellation that covers a given space without any gap or overlap.

\section{Optimal 3D Cell Association for Minimum LATENCY}

We now use the proposed 3D deployment model to refine our optimization problem in (6), as follows:

$$
\begin{aligned}
\min _{\mathcal{V}_{n}, n \in \mathcal{N}} & \sum_{n=1}^{N}\left[\int_{\mathcal{V}_{n}} \frac{\beta K_{n}}{B_{n} \log _{2}\left(1+\gamma_{n}(\boldsymbol{v})\right)} f(\boldsymbol{v}) \mathrm{d} \boldsymbol{v}+\frac{\beta K_{n}}{C_{n}}+g_{n}\left(\beta K_{n}\right)\right], \\
\text { s.t. } & K_{n}=L \int_{\mathcal{V}_{n}} f(\boldsymbol{v}) \mathrm{d} \boldsymbol{v}, \\
& \mathcal{V}_{l} \cap \mathcal{V}_{m}=\emptyset, \quad \forall l \neq m \in \mathcal{N}, \bigcup_{n \in \mathcal{N}} \mathcal{V}_{n}=\mathcal{V},
\end{aligned}
$$

where $\gamma_{n}(\boldsymbol{v})$ is the downlink SINR for a drone-UE located at $\boldsymbol{v}=(x, y, z)$ which is served by drone-BS $n$. Considering a practical bounded path loss model for air-to-air communications, the SINR can be given by:

$$
\gamma_{n}(\boldsymbol{v})=\frac{\eta P_{n}\left[1+d_{n}(\boldsymbol{v})\right]^{-\alpha}}{\sum_{u \in \mathcal{I}_{\text {int }}} \eta P_{u}\left[1+d_{u}(\boldsymbol{v})\right]^{-\alpha}+N_{o} B_{n}},
$$

where $\alpha$ is the path loss exponent, $N_{o}$ is the noise power spectral density, $\eta$ is the path loss constant. $d_{n}(\boldsymbol{v})$ is the distance of drone-BSs $n$ with a drone-UE located at $\boldsymbol{v}$. Also, $\mathcal{I}_{\text {int }}$ is the set of interfering drone-BSs.

Solving (10) is challenging since the optimization variables $\mathcal{V}_{n}, \forall n \in \mathcal{M}$, are continuous 3D association spaces which are mutually dependent. Furthermore, the fact that the size and shape of these 3D association spaces are unknown, exacerbates the complexity. In addition, the objective function in (10) does not have a closed-form expression thus making the problem intractable. Consequently, employing traditional optimization techniques (e.g., convex optimization) are not sufficient to solve (10). Here, we tackle our 3D space association by exploiting optimal transport theory, a mathematical tool that seeks an optimal mapping between two arbitrary probability measures. More specifically, in a semi-discrete optimal transport problem, a continuous probability density function must be mapped to a discrete probability measure.

Our cell association problem can be modeled as a semidiscrete optimal transport problem in which the source measure (drone-UEs' distribution) is continuous while the destination (distribution of drone-BSs) is discrete. Then, the optimal 3D cell partitions are obtained by optimally mapping the drone-UEs to drone-BSs. We note that, compared to our previous work in [10], this work is different in terms of the system model, the 3D cell association optimization problem, as well as the solution. Next, we characterize the solution of our optimization problem in (10).

Theorem 2. The optimal 3D cell association for drone-BS $l$, that leads to a minimum average latency in (10), is given by:

$$
\begin{aligned}
& \mathcal{V}_{l}^{*}=\left\{(x, y, z) \mid \alpha_{l}+\frac{K_{l}}{L} h_{l}(\boldsymbol{v})+\frac{\beta}{C_{l}}+g_{l}^{\prime}\left(\beta K_{l}\right)\right. \\
& \left.\leq \alpha_{m}+\frac{K_{m}}{L} h_{m}(x, y, z)+\frac{\beta}{C_{m}}+g_{m}^{\prime}\left(\beta K_{m}\right), \forall l \neq m\right\},
\end{aligned}
$$

where $h_{l}(\boldsymbol{v}) \triangleq \frac{\beta}{B_{n} \log _{2}\left(1+\gamma_{l}(\boldsymbol{v})\right)}$, and $\alpha_{l} \triangleq \int_{\mathcal{V}_{l}} h_{l}(\boldsymbol{v}) f(\boldsymbol{v}) \mathrm{d} \boldsymbol{v}$.

Proof. The existence of the optimal 3D cell partitions $\mathcal{V}_{n}$, $n \in \mathcal{N}$, can be verified by the existence of optimal transport maps [14]. Now, consider two 3D partitions $\mathcal{V}_{l}$ and $\mathcal{V}_{m}$, and a point $\boldsymbol{v}_{o}=\left(x_{o}, y_{o}, z_{o}\right) \in \mathcal{V}_{l}$. Also, let $B_{\epsilon}\left(\boldsymbol{v}_{o}\right)$ be a ball with a center $\boldsymbol{v}_{o}$ and radius $\epsilon>0$. Now, we generate the following new 3D partitions $\widehat{\mathcal{V}}_{n}$ :

$$
\left\{\begin{array}{l}
\widehat{\mathcal{V}}_{l}=\mathcal{V}_{l} \backslash B_{\varepsilon}\left(\boldsymbol{v}_{o}\right), \\
\widehat{\mathcal{V}}_{m}=\mathcal{V}_{m} \cup B_{\varepsilon}\left(\boldsymbol{v}_{o}\right), \\
\widehat{\mathcal{V}}_{n}=\mathcal{V}_{n}, \quad n \neq l, m
\end{array}\right.
$$

Let us define $p_{1}\left(K_{n}\right) \triangleq K_{n}, p_{2}\left(K_{n}\right) \triangleq \frac{\beta K_{n}}{C_{n}}, K_{\varepsilon}=$ $L \int_{B_{\varepsilon}\left(\boldsymbol{v}_{o}\right)} f(\boldsymbol{v}) \mathrm{d} \boldsymbol{v}$, and $\widehat{K}_{n}=L \int_{\widehat{\mathcal{V}}_{n}} f(\boldsymbol{v}) \mathrm{d} \boldsymbol{v}$. Considering the 
optimality of $\mathcal{V}_{n}, n \in \mathcal{N}$, we have:

$$
\begin{aligned}
& \sum_{n \in \mathcal{N}} \int_{\mathcal{V}_{n}} p_{1}\left(K_{n}\right) h_{n}(\boldsymbol{v}) f(\boldsymbol{v}) \mathrm{d} \boldsymbol{v}+p_{2}\left(K_{n}\right)+g_{n}\left(\beta K_{n}\right) \\
& \stackrel{(a)}{\leq} \sum_{n \in \mathcal{N}} \int_{\widehat{\mathcal{V}}_{n}} p_{1}\left(\widehat{K}_{n}\right) h_{n}(\boldsymbol{v}) f(\boldsymbol{v}) \mathrm{d} \boldsymbol{v}+p_{2}\left(\widehat{K}_{n}\right)+g_{n}\left(\beta \widehat{K}_{n}\right) .
\end{aligned}
$$

Canceling out the common terms leads to:

$$
\begin{aligned}
& \int_{\mathcal{V}_{l}} p_{1}\left(K_{l}\right) h_{l}(\boldsymbol{v}) f(\boldsymbol{v}) \mathrm{d} \boldsymbol{v}+p_{2}\left(K_{l}\right)+g_{l}\left(\beta K_{l}\right) \\
& +\int_{\mathcal{V}_{m}} p_{1}\left(K_{m}\right) h_{m}(\boldsymbol{v}) f(\boldsymbol{v}) \mathrm{d} \boldsymbol{v}+p_{2}\left(K_{m}\right)+g_{m}\left(\beta K_{m}\right) \\
& \leq \int_{\mathcal{V}_{m} \cup B_{\varepsilon}\left(\boldsymbol{v}_{o}\right)} p_{1}\left(K_{m}+K_{\epsilon}\right) h_{m}(\boldsymbol{v}) f(\boldsymbol{v}) \mathrm{d} \boldsymbol{v}+p_{2}\left(K_{m}\right) \\
& \quad+g_{m}\left(\beta\left(K_{m}+K_{\epsilon}\right)\right)+\int_{\mathcal{V}_{l} \backslash B_{\varepsilon}\left(\boldsymbol{v}_{o}\right)} p_{1}\left(K_{l}-K_{\epsilon}\right) h_{l}(\boldsymbol{v}) f(\boldsymbol{v}) \mathrm{d} \boldsymbol{v} \\
& \quad+p_{2}\left(K_{l}-K_{\epsilon}\right)+g_{l}\left(\beta\left(K_{l}-K_{\epsilon}\right)\right), \\
& \int_{\mathcal{V}_{l}}\left(p_{1}\left(K_{l}\right)-p_{1}\left(K_{l}-K_{\epsilon}\right)\right) h_{l}(\boldsymbol{v}) f(\boldsymbol{v}) \mathrm{d} \boldsymbol{v}+p_{2}\left(K_{l}\right)-p_{2}\left(K_{l}-K_{\epsilon}\right) \\
& +g_{l}\left(\beta K_{l}\right)-g_{l}\left(\beta\left(K_{l}-K_{\epsilon}\right)\right)+\int_{B_{\varepsilon}\left(\boldsymbol{v}_{o}\right)} p_{1}\left(K_{l}-K_{\epsilon}\right) h_{l}(\boldsymbol{v}) f(\boldsymbol{v}) \mathrm{d} \boldsymbol{v} \\
& \quad \leq \int_{\mathcal{V}_{m}}\left(p_{1}\left(K_{m}+K_{\epsilon}\right)-p_{1}\left(K_{m}\right)\right) h_{l}(\boldsymbol{v}) f(\boldsymbol{v}) \mathrm{d} \boldsymbol{v} \\
& \quad+p_{2}\left(K_{m}+K_{\epsilon}\right)-p_{2}\left(K_{m}\right)+g_{m}\left(\beta\left(K_{m}+K_{\epsilon}\right)\right)-g_{m}\left(\beta K_{m}\right) \\
& \quad+\int_{B_{\varepsilon}\left(\boldsymbol{v}_{o}\right)} p_{1}\left(K_{m}+K_{\varepsilon}\right) h_{m}(\boldsymbol{v}) f(\boldsymbol{v}) \mathrm{d} \boldsymbol{v}
\end{aligned}
$$

where $(a)$ comes from the fact that the sets $\mathcal{V}_{n}, \forall n \in \mathcal{N}$ are optimal 3D partitions and, thus, any variation of such optimal partitions, shown by $\widehat{\mathcal{V}}_{n}$, does not lead to a better solution. Note that, $K_{\epsilon}=L \int_{B_{\epsilon}\left(\boldsymbol{v}_{o}\right)} f(\boldsymbol{v}) \mathrm{d} \boldsymbol{v}$. Now, we multiply both sides of the inequality in (16) by $\frac{1}{K_{\epsilon}}$ and take the limit when $\epsilon \rightarrow 0$. After some algebraic manipulation, we can show that each optimal 3D cell association can be represented by:

$$
\begin{aligned}
\mathcal{V}_{l}^{*}= & \left\{(x, y, z) \mid \alpha_{l}+\frac{K_{l}}{L} h_{l} \boldsymbol{v}\right)+\frac{\beta}{C_{l}}+g_{l}^{\prime}\left(\beta K_{l}\right) \\
& \left.\leq \alpha_{m}+\frac{K_{m}}{L} h_{m}(\boldsymbol{v})+\frac{\beta}{C_{m}}+g_{m}^{\prime}\left(\beta K_{m}\right), \forall l \neq m\right\} .
\end{aligned}
$$

Using Theorem 2, we can determine the optimal 3D cell partitions associated with each drone-BS that ensure the minimum average latency for drone-UEs. From (14), we can see how the optimal 3D association depends on various network parameters such as the distribution of drone-UEs, locations of drone-BSs, backhaul data rate, load of the network, and the computational speed. Based on these parameters, Theorem 2 is utilized to optimally partition a specified space and determine a minimum latency 3D cell association scheme. In this case, to minimize the average latency, a drone-BS with a faster backhaul link and computational capabilities, or higher bandwidth and transmit power will serve more drone-UEs.

To solve (14), we propose the iterative algorithm shown in Algorithm 1. This algorithm, based on [14], can converge to the optimal solution within a reasonable number of iterations [14]. Algorithm 1 for solving (14) that finds the optimal 3D

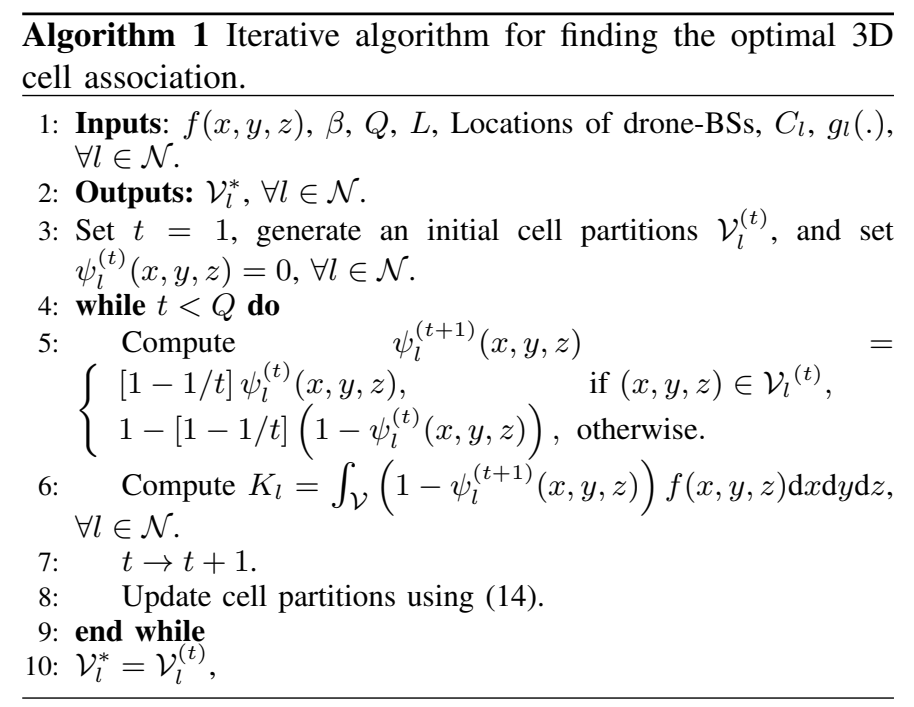

Table I: Simulation parameters.

\begin{tabular}{|c|c|c|}
\hline Parameter & Description & Value \\
\hline \hline$f_{c}$ & Carrier frequency & $2 \mathrm{GHz}$ \\
\hline$P_{n}$ & Drone-BS transmit power & $0.5 \mathrm{~W}$ \\
\hline$N_{o}$ & Noise power spectral density & $-170 \mathrm{dBm} / \mathrm{Hz}$ \\
\hline$L$ & Number of drone-UEs & 200 \\
\hline$B_{n}$ & Bandwidth for each drone-BS & $10 \mathrm{MHz}$ \\
\hline$\alpha$ & Path loss exponent & 2 \\
\hline$\eta$ & Path loss constant & $1.42 \times 10^{-4}$ \\
\hline$\beta$ & Packet size for drone-UE & $10 \mathrm{~kb}$ \\
\hline$C_{n}$ & Backhaul rate for drone-BS $n$ & $(100+n) \mathrm{Mb} / \mathrm{s}$ \\
\hline$\omega_{n}$ & Computation constant (i.e., speed) for each drone-BS & $10^{2} \mathrm{~Tb} / \mathrm{s}$ \\
\hline$\mu_{x}, \mu_{y}, \mu_{z}$ & Mean of the distribution & $(1000 \mathrm{~m}, 1000 \mathrm{~m}, 1000 \mathrm{~m})$ \\
\hline$\sigma_{x}, \sigma_{y}, \sigma_{z}$ & Standard deviation of the distribution & $(600 \mathrm{~m}, 600 \mathrm{~m}, 600 \mathrm{~m})$ \\
\hline
\end{tabular}

cell partitions proceeds as follows. The inputs are the 3D spatial distribution of drone UEs, number of drone-UEs, load, locations of the drone-BSs, computation time function, and the number of iterations, $Q$. First, we generate initial 3D cell partitions $\mathcal{V}_{l}^{(t)}$, and set $\psi_{l}^{(t)}(x, y, z)=0, \forall l \in \mathcal{N}$, with $\psi_{l}^{(t)}(x, y, z)$ being a pre-defined parameter which is used to update the cell partitions. Next, we update $\psi_{l}^{(t+1)}(x, y, z)$, and compute $K_{l}$ in step 6 . In step 8 , we update the partitions based on (14). Finally, we obtain the optimal 3D cell partitions and associations, at the end of the iteration.

\section{Simulation Results and Analysis}

For our simulations, we consider a cubic space of size $3 \mathrm{~km} \times 3 \mathrm{~km} \times 3 \mathrm{~km}$ in which 18 drone-BSs are deployed based on the proposed truncated octahedron approach to serve droneUEs. We determine the locations of drone-BSs by using (10) with parameters $a \in\{-1,0,1\}, b \in\{-1,0,1\}, c \in\{0,1\}$, and $R=400 \mathrm{~m}$. For drone-UEs' spatial distribution, we consider a 3D truncated Gaussian distribution. For the computation time, we consider a quadratic function of data size (i.e., load on each drone-BS), but our approach can accommodate any other arbitrary function. Here, the computation time for drone-BS $n$ is $g_{n}\left(\beta K_{n}\right)=\frac{\left(\beta K_{n}\right)^{2}}{\omega_{n}}$, with $\omega_{n}$ being the processing speed of drone-BS $n$. Unless stated otherwise, we use the simulation parameters listed in Table I. We compare our proposed 3D cell association with the classical SINRbased association (i.e., weighted Voronoi diagram) baseline.

Fig. 5 shows the average total latency as a function of the number of drone-UEs for the proposed 3D cell association and 


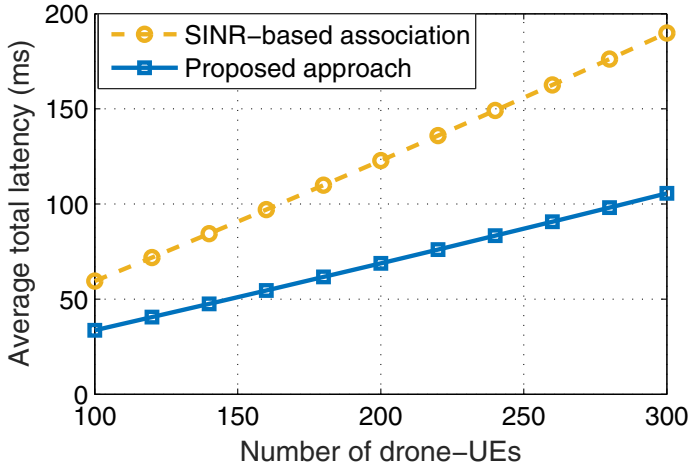

Fig. 5: Average total latency vs. number of drone-UEs.

the SINR-based association schemes. As we can see from this figure, the total latency increases by increasing the number of drone-UEs. A higher number of drone-UEs leads to a higher network congestion which, in turn, increases transmission time, backhaul latency, and computation time. Fig. 5 shows that, when the number of drone-UEs increases from 200 to 300 , the total latency increases by $56 \%$ and $42 \%$ for the SINRbased association and the proposed approach. Moreover, we can see that our proposed approach significantly reduces the latency compared to the SINR association case. This is due to the fact that, in our approach, besides SINR, the impact of congestion on the transmission, backhaul, and computational latencies is also taken into account. The proposed approach avoids creating highly congested 3D cell partitions that can cause excessive latency. From Fig. 5, we can see that our approach yields around $46 \%$ reduction in the average total latency compared to the SINR-based association.

Fig. 6 shows how the latency can be reduced by increasing the transmission bandwidth. By using more bandwidth, the transmission rate increases and, hence, the transmission latency decreases. Fig. 6 also reveals that our approach significantly enhances spectral efficiency compared to the SINRbased association. In essence, compared to the SINR case, the proposed approach requires less transmission bandwidth in order to meet a certain latency requirement. For instance, as we can see from Fig. 6, to ensure a $70 \mathrm{~ms}$ maximum total latency, our approach requires 57\% less bandwidth than the SINRbased association scheme. Another observation from Fig. 6 is that the rate of latency reduction decreases as the bandwidth increases. This is because in large bandwidth scenarios, the transmission latency can be smaller than the computation and backhaul latencies. Thus, the impact of decreasing the transmission latency on the total latency is relatively minor.

\section{CONCLUSION}

In this paper, we have introduced a novel framework for cell association and deployment in 3D cellular networks with drone-BSs and drone-UEs. We have proposed a tractable method for the 3D deployment of drone-BSs and solved the problem of cell association while minimizing the latency of drone users. In addition, based on the locations of droneBSs and drone-UEs' distribution, we have derived the optimal cell association of drone-UEs such that the latency for drone-

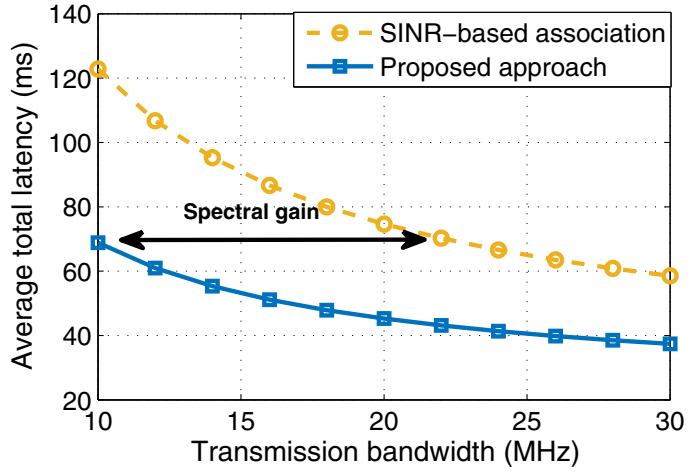

Fig. 6: Average total latency vs. transmission bandwidth.

UEs is minimized. Our results have shown that the proposed approach significantly reduces the latency of drone-UEs compared to the classical SINR-based association. Furthermore, the proposed latency-optimal cell association improves the spectral efficiency of the 3D drone-enabled wireless networks.

\section{REFERENCES}

[1] M. Mozaffari, A. T. Z. Kasgari, W. Saad, M. Bennis, and M. Debbah, "Beyond 5G with UAVs: Foundations of a 3D wireless cellular network," available online: arxiv.org/abs/1805.06532, 2018.

[2] I. Bor-Yaliniz and H. Yanikomeroglu, "The new frontier in RAN heterogeneity: Multi-tier drone-cells," IEEE Communications Magazine, vol. 54, no. 11, pp. 48-55, 2016.

[3] M. Mozaffari, W. Saad, M. Bennis, Y.-H. Nam, and M. Debbah, "A tutorial on UAVs for wireless networks: Applications, challenges, and open problems," available online: arxiv.org/abs/1803.00680, 2018.

[4] S. Zhang, Y. Zeng, and R. Zhang, "Cellular-enabled UAV communication: Trajectory optimization under connectivity constraint," in Proc. of IEEE International Conference on Communications (ICC), to appear, Kansas City, USA, May. 2018.

[5] M. Mozaffari, W. Saad, M. Bennis, and M. Debbah, "Efficient deployment of multiple unmanned aerial vehicles for optimal wireless coverage," IEEE Communications Letters, vol. 20, no. 8, pp. 1647-1650, Aug. 2016.

[6] M. Mozaffari, W. Saad, M. Bennis, and M. Debbah, "Unmanned aerial vehicle with underlaid device-to-device communications: Performance and tradeoffs," IEEE Transactions on Wireless Communications, vol. 15, no. 6, pp. 3949-3963, June 2016.

[7] M. Alzenad, A. El-Keyi, and H. Yanikomeroglu, "3-D placement of an unmanned aerial vehicle base station for maximum coverage of users with different QoS requirements," IEEE Wireless Communications Letters, vol. 7, no. 1, pp. 38-41, Feb. 2018.

[8] F. Lagum, I. Bor-Yaliniz, and H. Yanikomeroglu, "Strategic densification with UAV-BSs in cellular networks," IEEE Wireless Communications Letters, Early access, 2017.

[9] E. Kalantari, I. Bor-Yaliniz, A. Yongacoglu, and H. Yanikomeroglu, "User association and bandwidth allocation for terrestrial and aerial base stations with backhaul considerations," in Proc. IEEE Annual International Symposium on Personal, Indoor, and Mobile Radio Communications (PIMRC), Montreal, QC, Canada, Oct. 2017.

[10] M. Mozaffari, W. Saad, M. Bennis, and M. Debbah, "Wireless communication using unmanned aerial vehicles (UAVs): Optimal transport theory for hover time optimization," IEEE Transactions on Wireless Communications, vol. 16, no. 12, pp. 8052-8066, Dec. 2017.

[11] M. M. Azari, F. Rosas, A. Chiumento, and S. Pollin, "Coexistence of terrestrial and aerial users in cellular networks," in Proc. of IEEE Global Telecommunications Conference (GLOBECOM) Workshops, Singapore, Dec. 2017.

[12] S. Alam and Z. J. Haas, "Coverage and connectivity in three-dimensional networks," in Proc. of annual international conference on Mobile computing and networking, Los Angeles, CA, USA, Sep. 2006.

[13] H. S. M. Coxeter, Regular polytopes. Courier Corporation, 1973.

[14] G. Crippa, C. Jimenez, and A. Pratelli, "Optimum and equilibrium in a transport problem with queue penalization effect," Advances in Calculus of Variations, vol. 2, no. 3, pp. 207-246, 2009. 